operation would be slowed down further by more intermediate summary punch operations.

It can be shown that if $n_{0}$ is the order of the largest matrix which can be inverted given the storage capacity, and $n_{0}<n$, then the most efficient method of inverting by partitioning requires inverting of, say, $k$ submatrices, where the first $(k-1)$ matrices inverted are of order $n_{0}$, and the $k^{\text {th }}$ inverted matrix is of order $n-(k-1) n_{0}$.

HARVEY M. WAgner

The RAND Corporation

Santa Monica, Calif.

and

Stanford University

Stanford, Calif.

This article is contained in H. M. WAGNER, "Matrix Inversion on an Automatic Calculator by Row and Column Partitioning," P-417, The RAND Corporation, July 14, 1953; P-417 also discusses in detail (1) the inversion by partitioning of non-symmetric matrices; (2) optimal partitioning; (3) inversion of $n^{\text {th }}$ order matrices where $n$ exceeds storage limitations.

${ }^{1}$ W. Orchard-Hays, "The Duplex System for IBM's Model II CPC, A Fast Four Address, Double Operation, Floating-Decimal Set-Up," RM-1044, The RAND Corporation, February 23, 1953; H. M. WAGNER, "Coder's Manual for Duplex System" and "Stanford's Revised Duplex System-Wiring Manual," Technical Reports 1 and 4, Stanford Computation Center. These references give set-ups which meet the two operations per card requirement.

2 The formula and table following do not take into account any saving in cards and time at the beginning of the procedure when $r$ is small enough to invert without any summary punching. E.g., with 19 storage locations, a $5 \times 5$ matrix can be inverted without any summary punching in 90 cards at 150 cards/minute. In general, if $S$ is the number of storage locations available, then all $n^{\text {th }}$ order symmetric matrices for which $n \leqq N$ where $\frac{N^{2}+3 N-2}{2}=S$ can be inverted without any summary punching.

${ }^{3} \mathrm{H}$. Hotelling, "Some computational devices," Chapter X in Statistical Inference in Dynamic Economic Models, Cowles Commission Monograph 10, T. C. Koopmans, ed. New York, 1950, p. 323-328.

\title{
Polynomial Approximations to Elementary Functions
}

The increasing use of high-speed computing machines has revived interest in the approximation of functions of a real variable, particularly by polynomials. An orthodox table of function values at equidistant arguments may require considerable storage space in an electronic machine. In contrast, the coefficients of a polynomial which represents the function to a desired accuracy over a specified range may require very little storage space, and simple instructions will suffice for the evaluation of the polynomial.

If a function is bounded and continuous in a given finite range of argument, a powerful polynomial approximation is usually obtained by truncation of the infinite expansion of the function in Chebyshev polynomials. Many properties of these polynomials, and the means by which the coefficients in the infinite expansions can be derived, are given by Lanczos, ${ }^{1}$ whose notation for the Chebyshev polynomials in the range $0 \leq x \leq 1$ will be used here

$$
T_{n}{ }^{*}(x)=\cos n \theta, \quad \cos \theta=2 x-1 .
$$

In this note, tables are given which ease the calculation of polynomial approximations to some common functions. Coefficients in the infinite 
Chebyshev expansions valid in the specified ranges are given to nine decimal places, and formulae are also given for the Chebyshev polynomials.

A polynomial approximation to any of the functions considered is obtained by truncation of the infinite Chebyshev series. The retained coefficients are then rounded-off, and the polynomial rearranged in powers of the independent variable.

As an example, an approximation to $\sin \frac{1}{2} \pi x$ in the range $-1 \leq x \leq 1$ is

$$
x \sum_{n=0}^{5} A_{n} T_{n}^{*}\left(x^{2}\right),
$$

where the coefficients are given in the tables. Substituting their values gives

$$
\begin{aligned}
& \sin \frac{1}{2} \pi x \doteq x\left\{1.276278962-0.285261569\left(2 x^{2}-1\right)\right. \\
& \quad+0.009118016\left(8 x^{4}-8 x^{2}+1\right) \\
& \quad 0.00013 \quad 6587\left(32 x^{6}-48 x^{4}+18 x^{2}-1\right) \\
& \quad+0.000001185\left(128 x^{8}-256 x^{6}+160 x^{4}-32 x^{2}+1\right) \\
& \left.+0.000000007\left(512 x^{10}-1280 x^{8}+1120 x^{6}-400 x^{4}+50 x^{2}-1\right)\right\} \\
& \quad=1.570796326 x-0.645964102 x^{3} \\
& \quad+0.079692704 x^{5}-0.004681984 x^{7} \\
& \quad+0.000160640 x^{9}-0.000003584 x^{11}
\end{aligned}
$$

In this case, the rapidity of convergence of the Chebyshev series indicates that the truncation error will be small, and most of the error in the above approximation arises from the rounding-off of the coefficients $A_{n}$. Thus the maximum error cannot exceed three units in the ninth decimal place.

If an approximation is required to less than nine decimal accuracy, unwanted rounding errors can be avoided by retaining one or two extra decimals in the coefficients.

For instance, suppose that an approximation to $\ln (1+x)$ is required in the range $0 \leq x \leq 1$, accurate to three decimal places. Examination of the coefficients $\vec{A}_{n}$ in the series

$$
\ln (1+x)=\sum_{n=0}^{\infty} A_{n} T_{n}^{*}(x)
$$

given in the tables, shows that $\left|A_{n}\right|<0.0005$ when $n>3$, and that

$$
\sum_{n=4}^{\infty}\left|A_{n}\right|=0.000503 \cdots
$$

Hence

$$
\begin{aligned}
\ln (1+x) \doteq 0.37645+0.34315(2 x-1)-0.02944\left(8 x^{2}-8 x+1\right) & +0.00337\left(32 x^{3}-48 x^{2}+18 x-1\right) \\
= & 0.00049+0.98248 x-0.39728 x^{2}+0.10784 x^{3}
\end{aligned}
$$

with a maximum error not exceeding 0.00053 .

National Physical Laboratory

C. W. Clenshaw

Teddington, Middlesex

England

This note is published with the permission of the Director of the National Physical Laboratory.

${ }_{1}^{1}$ Tables of the Chebyshev Polynomials $S_{n}(x)$ and $C_{n}(x)$. NBS Applied Math. series 9, 1952. 
Tables of Chebyshev coefficients

$$
\begin{aligned}
& \sin \frac{1}{2} \pi x \\
& A_{n} \\
& 0 \quad 1.276278962 \\
& \begin{array}{lll}
1 & -0.28526 & 1569
\end{array} \\
& 0.009118016 \\
& -0.000136587 \\
& 0.000001185 \\
& 5 \quad-0.00000 \quad 0007 \\
& \sin \frac{1}{2} \pi x=x \sum_{n=0}^{\infty} A_{n} T_{n}{ }^{*}\left(x^{2}\right), \\
& -1 \leq x \leq 1 \text {. } \\
& \arctan x \\
& n \quad A_{n} \\
& \begin{array}{lll}
0 & 0.88137 \quad 3587
\end{array} \\
& \begin{array}{lll}
1 & -0.10589 & 2925
\end{array} \\
& 0.011135843 \\
& -0.001381195 \\
& 0.000185743 \\
& \begin{array}{lll}
5 & -0.00002 & 6215
\end{array} \\
& 6 \quad 0.000003821 \\
& \begin{array}{ll}
7 & -0.00000 \quad 0570
\end{array} \\
& 8 \quad 0.00000 \quad 0086
\end{aligned}
$$

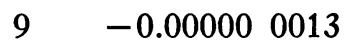

$$
\begin{aligned}
& 10 \quad 0.000000002 \\
& \arctan x=x \sum_{n=0}^{\infty} A_{n} T_{n}{ }^{*}\left(x^{2}\right), \\
& -1 \leq x \leq 1 \text {. } \\
& \text { for }|x|>1 \text {, use } \\
& \arctan x=\frac{1}{2} \pi-\arctan (1 / x) \\
& \arcsin x=x \sum_{n=0}^{\infty} A_{n} T_{n}{ }^{*}\left(2 x^{2}\right), \\
& -\frac{1}{2} \sqrt{2} \leq x \leq \frac{1}{2} \sqrt{2} \text {. } \\
& \arccos x=\frac{1}{2} \pi-x \sum_{n=0}^{\infty} A_{n} T_{n}{ }^{*}\left(2 x^{2}\right) \text {, } \\
& 0 \leq x \leq \frac{1}{2} \sqrt{2} \text {. } \\
& \arcsin x=\arccos \left(1-x^{2}\right)^{\frac{1}{2}} \text {, } \\
& \arccos x=\arcsin \left(1-x^{2}\right)^{\frac{1}{2}} \text {. }
\end{aligned}
$$

\begin{tabular}{ccc}
\multicolumn{3}{c}{$e^{x}$} \\
$n$ & \multicolumn{2}{c}{$A_{n}$} \\
0 & 1.75338 & 7654 \\
1 & 0.85039 & 1654 \\
2 & 0.10520 & 8694 \\
3 & 0.00872 & 2105 \\
4 & 0.00054 & 3437
\end{tabular}

\begin{tabular}{lrl} 
& \multicolumn{1}{c}{$e^{-x}$} & \\
$n$ & $A_{n}$ & \\
0 & 0.64503 & 5270 \\
1 & -0.31284 & 1606 \\
2 & 0.03870 & 4116 \\
3 & -0.00320 & 8683 \\
4 & 0.00019 & 9919
\end{tabular}




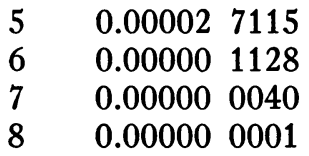

$e^{x}=\sum_{n=0}^{\infty} A_{n} T_{n}{ }^{*}(x), \quad 0 \leq x \leq 1$. $\log (1+x)$

$n \quad A_{n}$

$0 \quad 0.376452813$

$1 \quad 0.34314 \quad 5750$

$2-0.029437252$

$3 \quad 0.003367089$

$4-0.000433276$

$5 \quad 0.000059471$

$6-0.000008503$

$\begin{array}{llll}7 & 0.00000 & 1250\end{array}$

$8-0.000000188$

$9 \quad 0.000000029$

$10-0.000000004$

$11 \quad 0.000000001$ $\begin{array}{lll}5 & -0.00000 & 9975\end{array}$

$\begin{array}{lll}6 & 0.00000 & 0415\end{array}$

$\begin{array}{ll}7 & -0.00000 \\ 0015\end{array}$ $e^{-x}=\sum_{n=0}^{\infty} A_{n} T_{n}{ }^{*}(x), \quad 0 \leq x \leq 1$.

$$
\begin{array}{rrr}
\multicolumn{3}{c}{\Gamma(1+x)} \\
n & \multicolumn{1}{c}{A_{n}} \\
0 & 0.94178 & 5598 \\
1 & 0.00441 & 5381 \\
2 & 0.05685 & 0437 \\
3 & -0.00421 & 9835 \\
4 & 0.00132 & 6808 \\
& & \\
5 & -0.00018 & 9303 \\
6 & 0.00003 & 6069 \\
7 & -0.00000 & 6057 \\
8 & 0.00000 & 1056 \\
9 & -0.00000 & 0181 \\
& & \\
10 & 0.00000 & 0031 \\
11 & -0.00000 & 0005 \\
12 & 0.00000 & 0001
\end{array}
$$

$\log (1+x)=\sum_{n=0}^{\infty} A_{n} T_{n}{ }^{*}(x)$,

$$
0 \leq x \leq 1 \text {. }
$$

$$
J_{0}(x)
$$

n

$0 \quad 0.031540613$

$\begin{array}{llll}1 & -0.21461 & 6183\end{array}$

$2 \quad 0.004336620$

$3 \quad-0.266203654$

$4 \quad 0.306125520$

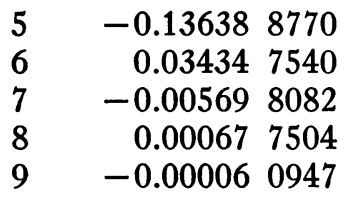

$\begin{array}{lr}10 \quad 0.00000 & 4309\end{array}$

$11-0.000000246$

$12 \quad 0.000000012$
$\Gamma(1+x)=\sum_{0=n}^{\infty} A_{n} T_{n}{ }^{*}(x)$,

$0 \leq x \leq 1$.

$$
J_{1}(x)
$$

$n$

$A_{n}$

0.0694243523

$-0.1155779057$

0.1216794099

$-0.1148840465$

0.0577905331

$-0.0169238801$

0.0032350252

$-0.0004370609$

0.0000440991

$-0.0000034583$

$10 \quad 0.00000 \quad 02172$

$11-0.0000000112$

$12 \quad 0.0000000005$ 


$$
\begin{aligned}
J_{0}(x) & =\sum_{n=0}^{\infty} A_{n} T_{n}^{*}\left(\frac{x^{2}}{100}\right), & J_{1}(x) & =x \sum_{n=0}^{\infty} A_{n} T_{n}^{*}\left(\frac{x^{2}}{100}\right), \\
& -10 \leq x \leq 10 . & & -10 \leq x \leq 10 .
\end{aligned}
$$

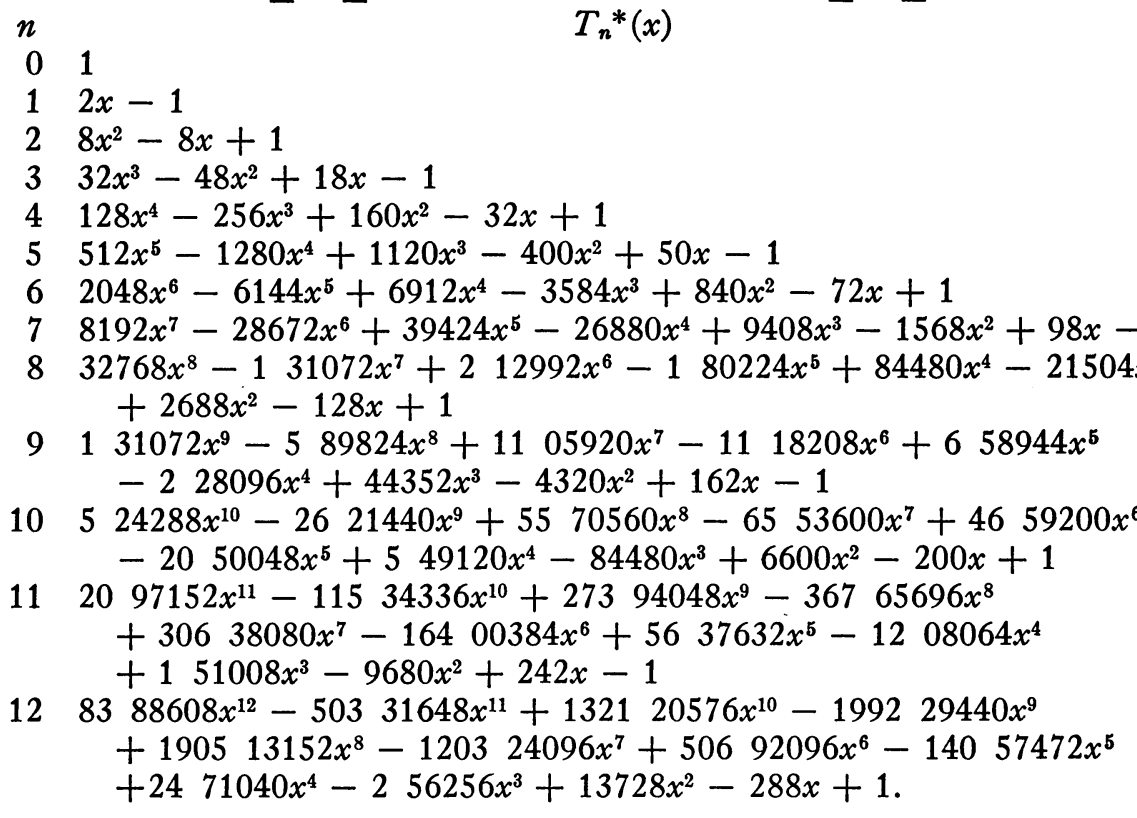

\section{RECENT MATHEMATICAL TABLES}

1202[A,P].-M. L. Clinnick, (a) Gear Ratios No. 43. (b) Gear Ratios No. 59. Privately printed, 3211 School Street, Oakland 2, California, 1953. Each book has 84 unnumbered pages $8.9 \times 11.4 \mathrm{~cm}$. and $8.26 \times 14.0 \mathrm{~cm}$. respectively, photo-offset from typescript. Price $\$ 1.00$ each.

These pocket-sized tables are designed for use in selecting appropriate sprocket gears in motorcycle racing events. They are triple entry tables giving $2 \mathrm{D}$ values of

$$
R=k r /(e c)
$$

for $c=10(1) 23, e=15(1) 25, r=46(1) 75, k=43,59$. Unrealistic values of $R \geq 15$ are omitted. In the intended application $r, e$ and $c$ are the numbers of teeth in the rear, engine, and countershaft sprockets respectively. The clutch sprocket is assumed to have 43 teeth or 59 corresponding to certain popular British and American makes of motorcycle. Instructions are given in (b).

$$
\text { D. H. L. }
$$

$1203[\mathrm{~A}, \mathrm{~B}, \mathrm{P}]$.- J. K. LyNCH, Kilocycle-Radian Frequency Conversion Tables. Commonwealth of Australia, Postmaster-general's Dept., Research Laboratories, Report No. 3726. Melbourne, 1953, 24 p. $20.2 \times 25.4 \mathrm{~cm}$.

This table gives $6 \mathrm{~S}$ values of

$$
\omega=2000 \pi F
$$

